\section{Die Macht des Codes}

Codes sind aus unserem Leben nicht mehr wegzudenken: Ohne Strichcode geht keine Ware mehr vom Stapel, ohne Code-Nummer sind unsere Kreditkarten wertlos und ohne AHV-Nummer bin ich nur noch ein halber Mensch. Der genetische Code beherrscht das reelle Leben und der binäre das virtuelle. Codes schützen meine Privatsphäre, Codes schützen auch Betrüger. Für Codes werden Kriege geführt, mit dem Knacken von Codes sind sie zu gewinnen. Codes verheissen Macht und Ansehen. Doch nur wenige entdecken den Stein von Rosette. Für die meisten von uns sind Codes nur langweilige Anhängsel des Lebens, Denkmäler des alltäglichen Einerleis.

In dieser Ausgabe befasst sich ein Artikel von Martin Denz [1] mit der Bedeutung der Codes in der Medizin. Ausgehend von der Besprechung eines Buches von $H$. R. Straub, "Das interpretierende
System", macht Denz deutlich, dass mit der Codierung von Daten im Gesundheitswesen nicht nur eine lästige und im Zunehmen begriffene Datenerfassung zu statistischen Zwecken erfolgt, sondern dass damit auch Grundlagen für Steuerungsprozesse in der Gesundheitspolitik gelegt werden. Damit bezeichnet er die Aufgabe der Codierung als eminent politische Frage und legt der Ärzteschaft nahe, diese Aufgabe als ihre ureigenste Verantwortung zu betrachten und nicht aus der Hand zu geben. Die Entscheidung über die Einführung und Verwendung von Klassifikationen und die Auswertung von Daten ist damit auch eine Frage der ärztlichen Berufsautonomie und eine der bedeutendsten Herausforderungen der nahen Zukunft. Denz' Überlegungen verdienen eine entsprechende Würdigung und eine breite Diskussion innerhalb der gesamten Ärzteschaft.

M. Trutmann

1 Denz MD. Medizinische Kodierung ist weit mehr als ein lästiges Übel! Schweiz Ärztezeitung 2002;83(8):375-8 